\title{
Cuando el trabajo aburre: Análisis de las propiedades psicométricas de la Escala de Aburrimiento Laboral (EAL)
}

\author{
Dr. Miguel Martínez Lugo y Dr. Ramón Rodríguez-Montalbán ${ }^{7}$ \\ Recibido: 02/05/2016 Aceptado: 19/07/2017 \\ DOI: $10.21772 /$ ripo.v35n1a01
}

\begin{abstract}
Resumen
El aburrimiento laboral es un fenómeno psicosocial que puede afectar a las personas en su trabajo y que se asocia con ambientes poco retadores y de baja estimulación. Para entender mejor este fenómeno, es necesario contar con instrumentos adecuados para su medición. La presente investigación tuvo el propósito de analizar las propiedades psicométricas de la Escala de Aburrimiento Laboral (EAL): validez de constructo, análisis de confiabilidad, análisis de los ítems, validez convergente y discriminante. Se realizaron tres estudios independientes con una muestra total de 2,032 participantes. El primer estudio evidencia que la EAL posee una estructura unifactorial y una confiabilidad apropiada. El segundo estudio provee evidencia de la validez convergente de la EAL con el Dutch Boredom Scale. El tercer estudio evidencia la validez discriminante de la EAL con la Escala de Engagement en el Trabajo de Utrecht. A la luz de los hallazgos, concluimos que la EAL es un excelente instrumento para medir el aburrimiento en el trabajo.
\end{abstract}

Palabras clave: Aburrimiento Laboral, Psicometría, Medición de Factores Psicosociales.

\section{When work is boring: Analysis of the psychometric properties of Job Boredom Scale (JBS)}

\begin{abstract}
Job boredom can be considered a psychosocial phenomenon that can affect people in their work environment, and that it's related with environments characterized by low challenges and low stimulation. To have a better understanding of the phenomenon of job boredom, an appropriate tool is necessary for its measurement. The aim of the present study is to analyze the psychometric properties of the Job Boredom Scale (JBS), particularly its construct validity, reliability, item analysis, convergent and discriminant validity. To do this, three independent studies were conducted with a total sample of 2,032 participants. The first study shows that the JBS has a single factor structure, and an appropriate reliability. The second study provides evidence of its convergent validity with the Dutch Boredom Scale. The third study provides evidence of its discriminant validity with the Utrecht Work Engagement Scale. The findings of these three studies lead us to conclude that the JBS is an excellent measure of job boredom.
\end{abstract}

Key words: Job Boredom, Psychometrics, Psychosocial Factors Measurement.

1 Universidad Carlos Albizu. Para ponerse en contacto con los autores lo puede hacer a las siguientes direcciones de correo electrónico: mmartinez@ albizu.edu y rmontalban@albizu.edu.

Los autores desean expresar su agradecimiento al Dr. Israel Sánchez-Cardona por sus aportaciones al desarrollo de la EAL. De igual forma queremos agradecer las recomendaciones de los dos revisores anónimos de la Revista Interamericana de Psicología Ocupacional.

Cómo citar este artículo: Martínez Lugo, M. \& Rodríguez-Montalbán, R. (2016). Cuando el trabajo aburre: Análisis de las propiedades psicométricas de la Escala de Aburrimiento Laboral (EAL). Revista Interamericana de Psicología Ocupacional, 35(1), 7-20. Doi: 10.21772/ ripo.v35n1a01 


\section{Introducción}

El aburrimiento laboral ha surgido en la literatura científica como una variable de interés que es importante estudiar ante los cambios económicos y organizacionales que hemos estado experimentando a lo largo del tiempo. Hoy día observamos personas que ocupan puestos de trabajo que no les retan, llevándolos a experimentar desinterés y poca estimulación. Por otro lado, los medios de comunicación populares también se han hecho eco de este interés, y presentan el aburrimiento laboral como una preocupación o riesgo para un ambiente de trabajo saludable.

Se han encontrado que el aburrimiento tienes múltiples consecuencias entre las que se encuentran: menor percepción de salud, mayor intención de abandono (van Hooff \& van Hooft, 2014), menor compromiso laboral (Reijseger et al, 2012), mayores conductas contraproducentes (Bruursema, 2007; van Hooff \& van Hooft, 2014), mayor ausentismo y menos satisfacción laboral (Kass, Vodanovich, \& Callender, 2011). Estos hallazgos evidencian que la experiencia del aburrimiento puede tener consecuencias negativas en el entorno laboral. Sin embargo, al momento, no encontramos instrumentos para estudiar la experiencia del aburrimiento en los entornos laborales latinoamericanos. ¿Cómo es posible entender la experiencia del aburrimiento en el contexto latinoamericano si no contamos con un instrumento para ello? ¿Cuáles son las propiedades psicométricas de la Escala de Aburrimiento Laboral (EAL)? ¿Están midiendo la misma experiencia en el trabajo la EAL y la Dutch Boredom Scale (DUBS)? ¿Es el aburrimiento laboral el opuesto empírico del engagement en el trabajo?

En este artículo pretendemos responder a estas interrogantes. Primero, desarrollamos un instrumento (EAL) para medir la experiencia del aburrimiento laboral en el contexto latinoamericano y analizamos sus propiedades psicométricas. Segundo, evidenciamos la validez convergente de la EAL y la DUBS. Por último, proveemos evidencia de que una persona no puede estar aburrida en el trabajo y engaged a la misma vez, ya que son experiencias psicológicamente incompatibles.

\section{Aburrimiento laboral}

Al revisar la literatura en la psicología industrial organizacional observamos diferentes momentos históricos en los cuales se han hecho planteamientos sobre el tema del aburrimiento en el trabajo. Encontramos que Smith (1944) en el Handbook of Industrial Psychology hace referencia a estudios realizados por Wyatt y Fraser en 1929 en los cuales se estudiaba el aburrimiento entre trabajadores que realizaban tareas repetitivas. En esta misma línea y en la década de 1960, Locke y Bryan (1967) encontraron que los objetivos de trabajo excesivamente fáciles tendían a provocar mayor aburrimiento. Por su parte, Hill (1975) una década más tarde encontró que el aburrimiento se relaciona negativamente con la extroversión y la edad. Décadas después, y en un nuevo siglo, van Wyk, De Beer, Pienaar y Schaufeli (2016) señalan que el aburrimiento es evidente cuando las capacidades del trabajador sobrepasan la complejidad de la tarea. Ante estos planteamientos presentados en diferentes momentos históricos cabe preguntarnos, ¿qué es el aburrimiento laboral?

\section{Definiciones y perspectivas}

La literatura científica presenta varias definiciones para explicar qué es el aburrimiento en el trabajo. Gemmill y Oakley (1992) establecen que el aburrimiento ocurre en el ambiente laboral cuando los empleados son incapaces de experimentar emocional e intelectualmente un significado personal en lo que están haciendo en su proceso de trabajo. Fisher (1993), por su parte, se refiere al aburrimiento como un estado afectivo no placentero y pasajero en el cual el individuo siente una falta de interés y dificultad para concentrarse en una actividad. De igual manera, O'Hanlon (1981) conceptúa el aburrimiento como un 
estado psicofísico único que es producido por la exposición prolongada a una situación monótona. Para Mikulas y Vodanovich (1993) el aburrimiento es un estado de excitación relativamente bajo y de insatisfacción que se atribuye a un ambiente inadecuadamente estimulante. De igual forma, Loukidou, Loan-Clarke y Daniels (2009) establecen que el aburrimiento laboral es una respuesta a un trabajo pasivo, poco retador y no placentero. Fahlman, Mercer-Lynn, Flora y Eastwood (2011) indican que el aburrimiento laboral es una experiencia aversiva relacionada con el tener un deseo no llenado de estar involucrado en una actividad que satisfaga. En la misma línea de los autores antes citados, van Hooff y van Hooft (2014) conceptúan el aburrimiento como un estado emocional negativo y desactivador que surge al realizar actividades laborales.

Comoobservamos, en muchas delas definiciones se presenta el aburrimiento como una experiencia negativa, relacionada con la insatisfacción, de bajo nivel de activación, que lleva a percibir la situación como carente de significado (Kass, Vodanovich, \& Callender, 2001; van Hooff \& van Hooft, 2014; Vodanovich \& Watt, 2016). Se conceptúa además el aburrimiento como un estresor o un aspecto del ambiente del trabajo que lleva o genera emociones negativas (Bruursema, Kessler \& Spector, 2011).

De la misma manera que existen varias definiciones del aburrimiento laboral, la literatura presenta dos conceptuaciones principales del constructo (Reijseger et al, 2012). La primera, y la más tradicional, identifica el aburrimiento asociado a la realización de tareas monótonas y repetitivas. Esto se conoce como aburrimiento situacional y se relaciona con variables afectadas por el ambiente en el cual se desempeña el individuo (Kass et al., 2001).

En esta primera perspectiva se establece que el aburrimiento surge por factores externos al individuo como son las condiciones de contexto o ambiente y las variables asociadas a las tareas que la persona debe desempeñar (Sohail, Ahmad,
Tanveer \& Tarik, 2012). Los primeros estudios del aburrimiento, indicaban que este era causado por una baja estimulación externa asociada a un trabajo monótono (Locke \& Bryan, 1967; O’Hanlon, 1981).

Apoyando este primer acercamiento, varios autores establecen que el aburrimiento laboral se asocia a características de la tarea como son la baja carga de trabajo y la monotonía (Van der Heijden, Schepers \& Nijssen, 2012; van Wyk et al., 2016), la ausencia de variedad en la tarea, las oportunidades limitadas de aprendizaje (Guglielmi, Simbula, Mazzetti, Tabanelli \& Bonfiglioli, 2013), la deficiencia en las tareas que hay que realizar (Mikulas \& Vodanovich, 1993) y alta burocracia y mecanismos de control (Harju, Hakanen \& Schaufeli, 2014). Csikszentmihalyi (1975) añade que el aburrimiento laboral se experimenta cuando las habilidades de los empleados exceden las demandas laborales.

Por otra parte, la segunda conceptuación del aburrimiento indica que este es una disposición o un rasgo personal. Según esta perspectiva, el aburrimiento rasgo tiene sus orígenes en el individuo y se asocia a factores como la personalidad, la inteligencia y la salud mental (Sohail et al, 2012). Los exponentes de esta perspectiva establecen que las personas propensas al aburrimiento experimentan depresión, desesperanza, soledad y la tendencia a distraerse con facilidad (Bruursema, 2007). Realizar una tarea común requiere, para estas personas, de mucho esfuerzo llevándolos a experimentar insatisfacción con lo que se hace y con su bienestar psicológico (Farmer \& Sundberg, 1986). De acuerdo a esta perspectiva, las personas aburridas demuestran además niveles bajos de ambición, prudencia, sociabilidad, éxito (Watt, 2002) y menor satisfacción con diferentes aspectos de su trabajo (Kass et al., 2001). Se indica en la literatura que aquellas personas con una predisposición al aburrimiento no regulan bien sus emociones mostrando niveles altos de coraje y agresividad, están menos 
abiertos a nuevas experiencias, son menos sociables, menos agradables, menos organizados y tienden a manipular más a las otras personas en sus relaciones sociales (Culp, 2006). Desde el punto de vista del trabajo, Bruursema (2007) encontró una asociación entre el aburrimiento rasgo y los comportamientos organizacionales contraproducentes, presentando así evidencia de cómo incide este en los comportamientos a nivel organizacional.

\section{Manifestaciones del aburrimiento}

El aburrimiento se manifiesta de diferentes formas en los contextos de trabajo. van Hooff y van Hooft (2014) denominan estas manifestaciones, los comportamientos de aburrimiento y los definen como aquellos comportamientos específicos de alejamiento que realizan los trabajadores y que no son funcionales para la obtención de las metas del trabajo. Entre las manifestaciones más frecuentes del aburrimiento que se mencionan en la literatura se encuentran la falta de atención, soñar despierto, tener pensamientos no relacionados al trabajo, tener una percepción distorsionada del pasar del tiempo y realizar tareas no relacionadas a las exigencias de su rol laboral (Reijseger et al., 2012). Relacionado a este último punto, existe evidencia que señala que cuando el empleado se aburre, se involucra en actividades no relacionadas al trabajo como puede ser leer revistas, comer, conversar sobre temas no relacionados al trabajo, jugar con su celular/móvil, mirar por la ventana, hacer dibujos y garabatos, hacer chistes, tomar café/té, enviar e-mails personales y usar el internet para asuntos personales (Baker, 1992; Martin, Sadlo, \& Stew, 2006).

Harju et al. (2014) señalan que se ha realizado muy poca investigación académica sobre el aburrimiento laboral, su ocurrencia en diferentes contextos de trabajo y cómo se asocia con diferentes resultados. Piotrowski (2013) realizó una búsqueda en PsycINFO que cubrió los años de 1923 a 2013 y encontró un total de 312 títulos de artículos sobre el tema de aburrimiento. Esto llevó a Piotrowski a resaltar la necesidad de integrar el aburrimiento como una variable moderadora en la investigación en Psicología Industrial/ Organizacional. Recientemente Vodanovich y Watt (2016) realizaron una nueva búsqueda para junio de 2015 e identificaron 725 referencias en el tema, destacando que las investigaciones más frecuentes giran en torno a las diferencias individuales en el aburrimiento y a su medición y evaluación.

Revisamos la literatura sobre el tema de aburrimiento laboral y hallamos estudios recientes como el realizado por Harju et al. (2014) quienes encontraron una asociación entre el aburrimiento y las autoevaluaciones pobres del estado de salud, la presencia de más síntomas de estrés, mayor intención de abandono de la organización y una mayor intención de jubilarse prematuramente. Por su parte, van Hooff y van Hooft (2014) encontraron relaciones negativas entre el aburrimiento laboral y la edad, el aumento en recursos laborales estructurales y la disminución en las demandas retadoras de trabajo. Estos autores también encontraron relaciones positivas entre el aburrimiento laboral, el distrés y los comportamientos laborales contraproducentes. En la misma línea, Alda et al., (2015) encontraron correlaciones positivas entre el aburrimiento laboral y el afecto negativo y la ansiedad. En varias investigaciones también se evidencia la relación entre el aburrimiento y la depresión (Alda et al., 2015; van Hooff \& van Hooft, 2014; van Hooff \& van Hooft, 2016).

En diferentes publicaciones se ha señalado y evidenciado la relación del aburrimiento laboral con diferentes variables organizacionales. Entre estas se incluyen las siguientes: pobre desempeño, accidentes y heridas, ausentismo, errores en el trabajo, abuso de drogas y alcohol, comportamiento de trabajo contraproducentes, bajo compromiso organizacional, estrés, insatisfacción laboral, depresión, ausencias por enfermedad, intención de abandono y percepción pobre de salud (Bruursema 
et al., 2011; Harju et al., 2014; Kass et al., 2001; Loukidou et al., 2009; Mann, 2012; van Hooff \& van Hooft, 2014; Schaufeli \& Salanova, 2014). Recientemente se han encontrado relaciones negativas del aburrimiento con el engagement en el trabajo y el compromiso organizacional (Harju et al., 2016; van Wyk et al., 2016).

\section{Medición del aburrimiento}

Para poder avanzar en la investigación del aburrimiento es necesario tener instrumentos para medir dicha variable (Harju et al., 2014). $\mathrm{Al}$ revisar la literatura existente encontramos que se han desarrollado diferentes instrumentos cuyo propósito es obtener información sobre el aburrimiento (para una presentación más abarcadora sobre este tema vea a Vodanovich, 2003 y a Vodanovich \& Watt, 2016). Como presenta Vodanovich, en muchas investigaciones se ha utilizado solo un ítem para medir el aburrimiento (Ambrose et al.; Daniels; Fisher; Melamed et al; Spector et al.; Yang \& Diefendorff). En otras, se han desarrollado instrumentos más extensos y que responden a las conceptuaciones principales antes mencionadas (aburrimiento como rasgo o aburrimiento situacional). Por ejemplo, en 1975, Grubb desarrolló Job Boredom Scale (JBS) que tiene 11 ítems que se responden en un formato de respuesta de 5 puntos y que se agrupan en dos sub-escalas: cognitiva y afectiva. En 1986 aparece el Lee Job Boredom Scale (LJBS) que tiene 17 ítems en un formato de respuesta de 5 puntos. En dicho año también surgió el Boredom Proneness Scale (BPS) de Farmer y Sunberg. Esta escala tiene 28 ítems que se responden en un formato de cierto o falso. En 2011 Fahlman et al. desarrollaron el Multidimensional State Boredom Scale (MSBS) que tiene 29 ítems en un formato de 7 puntos agrupados en 5 sub-escalas: desvinculación, alta estimulación, falta de atención, baja estimulación, percepción del tiempo. La última escala encontrada en la literatura revisada es la desarrollada por Reijseger et al. en 2012 y que se conoce como Dutch Boredom Scale (DUBS). Esta tiene 6 ítems que se responden en un formato de 5 puntos. De todos los instrumentos reseñados por Vodanovich y Watt (2016), solo dos están dirigidos a medir el aburrimiento en el contexto laboral: Lee Job Boredom Scale y Dutch Boredom Scale.

Tal y como se evidencia, no se han desarrollado escalas para medir el aburrimiento laboral en el idioma español que nos ayuden a investigar este constructo de la manera más adecuada, contribuyendo así en la investigación, identificación y prevención de este constructo. Solo se identificó un estudio donde se tradujo y validó la MSBS en una muestra en España (Alda, et al., 2015). Es por esto que el objetivo de este trabajo es presentar los resultados relacionados con el desarrollo y validación de la Escala de Aburrimiento Laboral (EAL) de Martínez-Lugo, Rodríguez-Montalbán y Sánchez-Cardona.

Para el desarrollo de la escala y sus reactivos, los autores de la misma definieron el aburrimiento laboral como un estado subjetivo relacionado al trabajo compuesto por la desvinculación, el desinterés y la percepción de la prolongación del tiempo en el trabajo. La desvinculación se conceptúa como el distanciamiento psicológico de las tareas y/o actividades relacionadas en el trabajo causado por una falta de estimulación o reto asociada a la naturaleza del trabajo. El desinterés, por su parte, se define como un sentimiento de indiferencia ante las tareas y/o actividades que se realizan. Por último, la distorsión de la percepción del tiempo implica percibir una excesiva prolongación del tiempo en el lugar de trabajo, que el tiempo pasa muy lento. Esta definición tomó como base la literatura revisada asociada con las perspectivas, manifestaciones y resultados del aburrimiento laboral que presentamos previamente.

\section{Aburrimiento laboral y engagement en el trabajo: ¿ Dos posibles opuestos?}

La investigación del engagement en el trabajo surge como corolario al estudio del síndrome de quemarse por el trabajo (SQT) (RodríguezMontalbán, Vélez-Pastrana \& Meléndez-Sáez, 
2012). Para Schaufeli y Salanova (2007) el engagement es un estado psicológico considerado la antítesis positiva del SQT. Schaufeli, Salanova, González-Romá y Bakker (2002) definen el engagement como "un estado mental positivo de realización relacionado con el trabajo caracterizado por el vigor, la dedicación y la absorción” (p. 74). El vigor se caracteriza por tener altos niveles de energía y resistencia mental mientras se trabaja y por el deseo de esforzarse aun cuando se presentan dificultades. Por su parte, la dedicación se refiere a un alto nivel de implicación, sentido, entusiasmo, inspiración, orgullo y reto por el trabajo. Por último, la absorción se produce cuando una persona está completamente concentrada en lo que hace, teniendo un alto nivel de disfrute, experimentando dificultades para desconectarse y sintiendo que el tiempo pasa rápidamente.

Entendemos que el aburrimiento es una experiencia psicológica opuesta al engagement debido a que una persona no puede experimentar al mismo tiempo altos niveles de energía (vigor), entusiasmo (dedicación) y sentir que el tiempo pasa rápido (absorción) mientras está aburrida. González-Romá, Schaufeli, Bakker y Lloret (2006) evidenciaron que el engagement y el SQT constituyen dos dimensiones psicológicas diferentes. Por lo que el que una persona no esté engaged en el trabajo no es sinónimo de que esté quemada en el mismo. Por lo antes expuesto, proponemos que una persona engaged no puede estar aburrida al mismo tiempo. Es por esto que nuestra propuesta es que el aburrimiento es el opuesto psicológico del engagement en el trabajo.

\section{Propósito del estudio}

En términos específicos, nuestro estudio tiene los siguientes objetivos:

1. Analizar la estructura factorial de la EAL.

2. Analizar la confiabilidad interna de la EAL.

3. Analizar la validez convergente y discriminante de la EAL.

\section{Plan de la investigación}

Para cumplir con los objetivos de la presente investigación se realizaron tres estudios empíricos con tres muestras independientes. Cada uno de estos estudios va dirigido a atender los diferentes objetivos planteados anteriormente. En el primer estudio se analizaron las propiedades psicométricas de la EAL (validez de constructo, análisis de confiabilidad y análisis de los ítems). En el segundo estudio se realizó un análisis de validez convergente entre la EAL y el Dutch Boredom Scale (DUBS). Por último, en el tercer estudio se realizó un análisis de validez discriminante de la EAL con la Escala de Engagement en el Trabajo de Utrecht (UWES-9 por sus siglas en inglés).

\section{Método general}

\section{Procedimiento general}

Los tres estudios contaron con la aprobación del Comité de Ética Institucional (IRB por sus siglas en inglés) de la Universidad Carlos Albizu. Los participantes de estas investigaciones fueron contactados de forma individual. Como criterios de inclusión para participar en los diferentes estudios se estableció que la persona debería tener 21 años o más, estar trabajando al momento del estudio y saber leer y escribir en castellano. A cada persona se le entregó un documento de consentimiento informado, en el cual se le informaba el propósito del estudio y se le garantizaban sus derechos como participantes. Una vez la persona accedía a participar, se le entregaban los cuestionarios para que los completara.

\section{Análisis de datos general}

Los datos de cada participante en cada estudio fueron entrados en una plantilla de datos para su posterior análisis. En el Estudio 1 los datos fueron sometidos a análisis de correlación, análisis de factores exploratorio, análisis confirmatorio de factores con ecuaciones estructurales y análisis de confiabilidad. En el Estudio 2 se realizó un análisis confirmatorio de factores de los instrumentos EAL 
y DUBS. También se realizó un análisis de varianza media extraída, análisis de confiabilidad de ítems y factores latentes, análisis de correlación y análisis de validez convergente. Por último, en el Estudio 3 se realizó un análisis de correlación $r$ de Pearson y un análisis de correlación entre factores latentes. Para realizar estos análisis se utilizaron los programas estadísticos IBM SPSS 23 y AMOS 23.0.

\section{Estudio 1 Desarrollo del instrumento}

\section{Participantes}

$\mathrm{El}$ análisis de la estructura factorial de la EAL se realizó con una muestra por disponibilidad de 1,049 trabajadores en Puerto Rico. Los datos fueron recopilados durante los meses de enero a abril de 2011. Los participantes en su mayoría eran mujeres (61.2\%), con una edad promedio de 36.85 años $(D E=10.87)$ y el $43.1 \%$ informó tener una preparación académica a nivel de bachillerato (licenciatura). La muestra provino en su mayoría (75\%) de empresas privadas dedicadas al área de servicio (54.6\%). El 73\% de los participantes informó tener un empleo regular. La muestra informó poseer una media de 14.38 años de experiencia en su trabajo $(D E=10.38)$.

\section{Instrumentos}

Para obtener los datos referentes a las variables del estudio, los investigadores administraron los siguientes instrumentos:

Cuestionario de datos generales. En este cuestionario se solicitó información referente al género sexual, edad, preparación académica, estado civil, número de años trabajando, tipo de organización donde trabaja, tipo de industria para la cual trabaja, número de horas trabajadas a la semana y tipo de empleo que tiene con la organización.

Escala de Aburrimiento Laboral (EAL). Esta escala fue desarrollada por Martínez- Lugo, Rodríguez-Montalbán y Sánchez-Cardona. La escala administrada tenía un total de 10 ítems a los cuales se responde en un formato de respuesta que fluctúa desde 0 (totalmente en desacuerdo) a 6 (totalmente de acuerdo). Para el desarrollo de la escala se tomó como base la definición del constructo desarrollada por los autores y que fue presentada anteriormente. Los 10 ítems se distribuyen en las tres sub-escalas: desvinculación, desinterés y percepción temporal.

\section{Resultados del Estudio 1}

Se realizó un análisis de factores exploratorio y se encontró que los 10 ítems se ubicaron en un solo factor el cual explica el $72 \%$ de la varianza. Luego se procedió a realizar un análisis confirmatorio partiendo de la estructura de tres factores sugerida por los autores (desvinculación, desinterés y percepción temporal). Este modelo de tres factores presenta un buen ajuste a los datos $\left(X^{2}(32) 284.71\right.$, $p<.001 ; G F I=95 ; A G F I=.91 ; C F I=.97 ; N F I=.97$; $T L I=.96 ; I F I=.97 ;$ RMSEA $=.08$ ), sin embargo las correlaciones entre los factores son demasiado altas (.88 a .98), lo que sugiere que hay mucha varianza compartida entre los factores (ver Figura 1). Dados estos resultados, optamos por poner a prueba el modelo de un factor latente de aburrimiento.

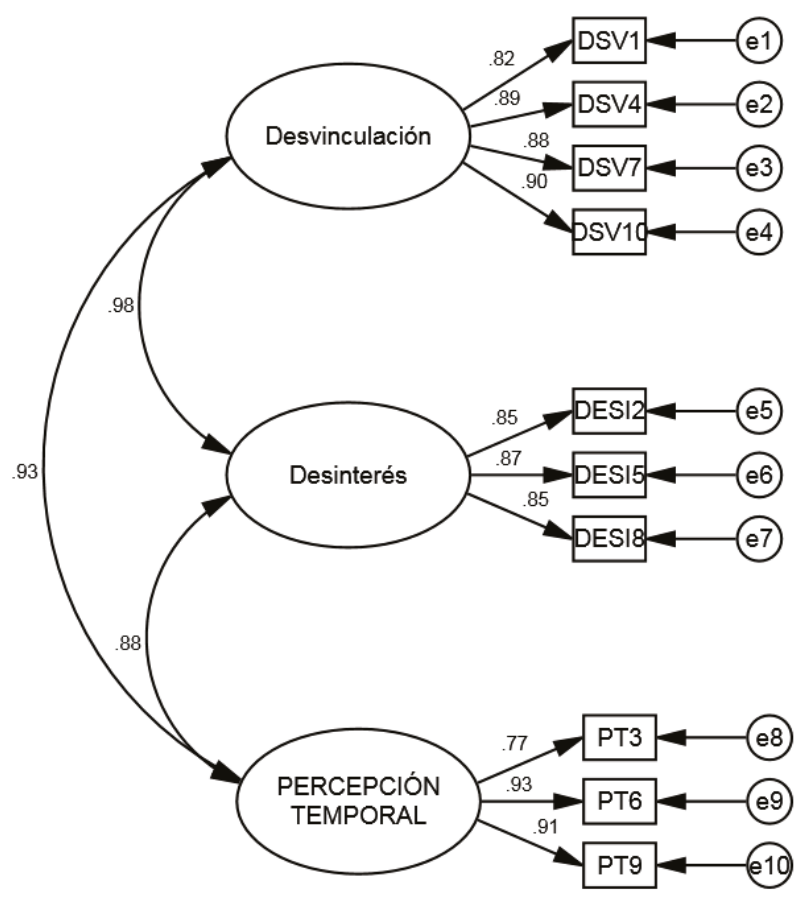

Figura 1: Modelo de tres factores 
Al realizar el análisis, encontramos que el modelo de un solo factor de aburrimiento no posee buenos índices de ajuste $\left(X^{2}(35) 598.22\right.$, p <.001; $\quad G F I=89 ; \quad A G F I=.83 ; \quad C F I=.94$; $N F I=.94 ; T L I=.93 ; I F I=.94 ; R M S E A=.12$ ), por lo que realizamos un análisis de los índices de modificación de los errores (ver Figura 2). Además, siguiendo un criterio cualitativo y cuantitativo se observó que hay redundancia en los ítems, por tanto eliminamos los ítems con menor carga factorial. Una vez eliminados los dos ítems (Las tareas que realizo en mi trabajo carecen de sentido para mi; Siento que el tiempo pasa lentamente en mi trabajo) el modelo mejoró significativamente.

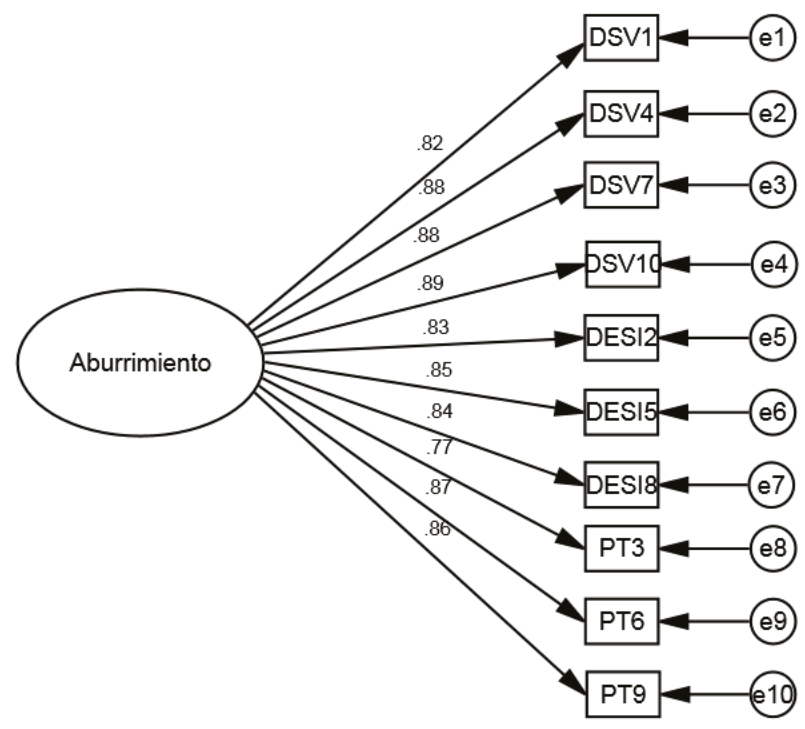

Figura 2: Modelo inicial de un factor

Este modelo final presenta mejores índices de ajuste $X^{2}$ (20) 152.346, $p<.001 G F I=96$; $A G F I=.93 ; C F I=.98 ; N F I=.98 ; T L I=.97 ; I F I=.98$; $R M S E A=.07$ y es más parsimonioso (véase Figura 3). Tomando esta información como base se decidió que la versión final de la EAL tiene ocho ítems.

Por último, se realizó un análisis de confiabilidad de la forma final de la EAL y se obtuvo un coeficiente alfa de Cronbach de .95 y la fiabilidad compuesta es de .96. Por otro lado, la correlación ítem-total promedio de los ítems fue de .83. Se analizó también la Varianza Media Extraída (AVE) y se obtuvo un valor de .75 .

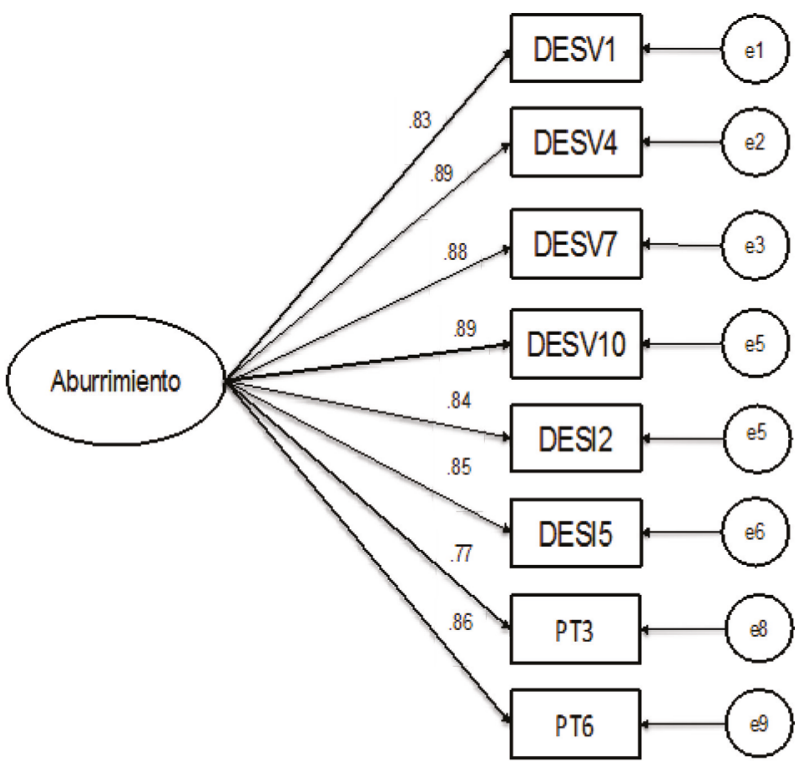

Figura 3: Modelo final para la EAL

\section{Estudio 2 Validez convergente}

\section{Participantes}

Para estudiar la validez convergente de la EAL se contactó una muestra por disponibilidad de 583 trabajadores en Puerto Rico. Los datos fueron recopilados durante los meses de enero a abril de 2015. Los participantes en su mayoría eran mujeres (57.1\%), con una edad promedio de 35.49 años $(D E=11.45)$. El $47.7 \%$ informó tener una preparación académica a nivel de bachillerato (licenciatura). La muestra provino en su mayoría (71\%) de empresas privadas dedicadas al área de servicio (39\%). El 76\% de los participantes informó tener un empleo regular. La muestra informó poseer una media de 13.22 años de experiencia en su trabajo $(D E=10.55)$.

\section{Instrumentos}

Para obtener los datos referentes a las variables del estudio, los investigadores administraron los siguientes instrumentos que ya fueron descritos en el estudio anterior: Cuestionario de datos generales y la Escala de Aburrimiento Laboral (EAL) de 8 ítems.

Además se utilizó Dutch Boredom Scale (DUBS) (versión en español) desarrollada por Reijseger et al. (2012). Esta escala tiene 6 ítems y 
los análisis psicométricos realizados por sus autores indican que la misma posee un factor que explica el $51 \%$ de la varianza y tiene un índice de consistencia interna alfa de Cronbach de .80. El promedio de las correlaciones ítem total fue de .55 .

\section{Resultados del Estudio 2}

Primeramente se realizó un análisis de correlación entre las puntuaciones en la EAL y DUBS, encontrándose un coeficiente de correlación de Pearson de .81. Luego se llevó a cabo un análisis confirmatorio de factores con ecuaciones estructurales donde se correlacionaron como dos factores latentes cada una de las escalas $X^{2}$ (76) $528.226 p<.001, C F I=.95, N F I=.93$, $T L I=.93, I F I=.95, R M S E A=.08$. Los coeficientes de regresión de la EAL fluctuaron entre $.72 \mathrm{y}$ .88 , mientras que los coeficientes de regresión de DUBS fluctuaron entre .60 y .90. La correlación entre los dos factores latentes es de .93. Cabe destacar que la varianza compartida entre ambos instrumentos resultó de .87 . Esto es indicativo de que ambas escalas están midiendo el mismo constructo (Kline, 2005).

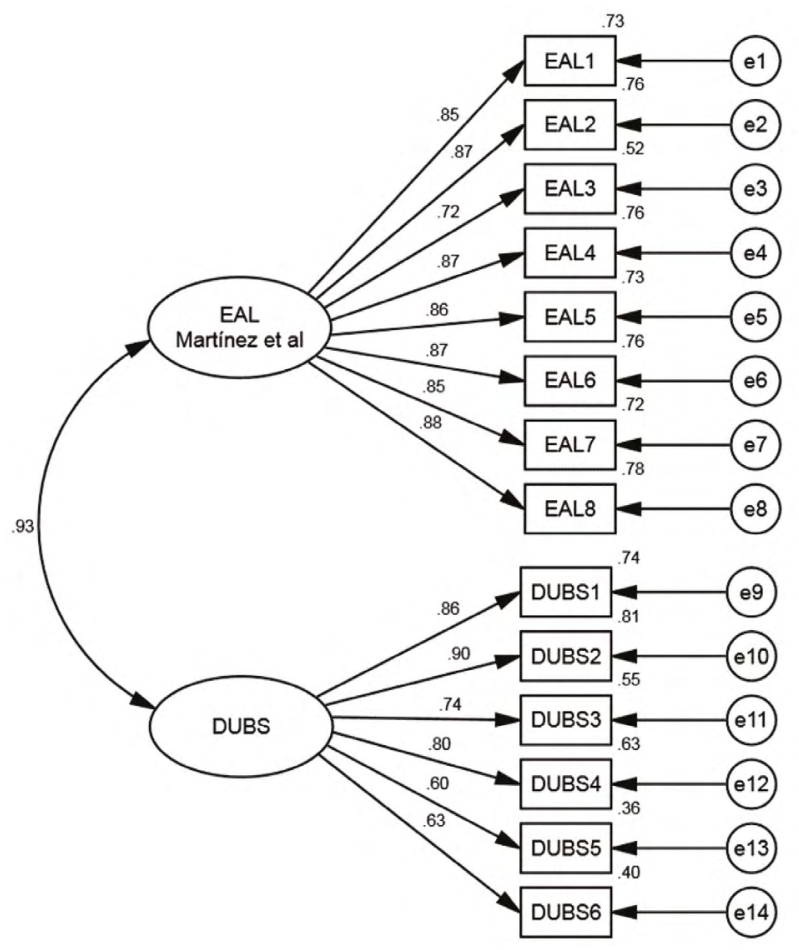

Figura 4: Modelo de validez convergente EAL y DUBS
Luego se procedió a calcular la varianza media extraída para las escalas y se obtuvieron los siguientes resultados: $\mathrm{EAL}=.72$ y DUBS $=.58$. Por otra parte, se calculó la fiabilidad compuesta de ambas escalas obteniéndose un valor de .95 para EAL y .89 para DUBS. Por último, se realizó un análisis de confiabilidad obteniéndose un coeficiente alfa de Cronbach de .95 para EAL y de .89 para el DUBS.

\section{Estudio 3 Validez discriminante}

\section{Participantes}

El análisis de validez discriminante se realizó con una muestra por disponibilidad de 400 trabajadores en Puerto Rico. Los datos fueron recopilados durante los meses de enero a abril de 2012. Los participantes en su mayoría eran mujeres (62.3\%), con una edad promedio de 36.06 años $(D E=10.79)$ y una media de 13.24 años de experiencia en su trabajo $(D E=10.47)$. El $41.6 \%$ informó tener una preparación académica a nivel de bachillerato (licenciatura). El 67.1\% de los participantes informó tener un empleo regular. La muestra en su mayoría (75.9\%) indicó que labora en empresas privadas dedicadas al área de servicio (54.6\%).

\section{Instrumentos}

Para obtener los datos referentes a las variables del estudio, los investigadores administraron los siguientes instrumentos que ya fueron descritos anteriormente: Cuestionario de datos generales y la Escala de Aburrimiento Laboral (EAL) de 8 ítems.

Para obtener los datos pertinentes para este estudio se administró también la Escala de Engagement en el Trabajo de Utrecht (UWES-9 por sus siglas en inglés) desarrollada por Shaufeli, Bakker y Salanova (2006). Esta escala tiene 9 ítems que se responden en una escala de 7 anclajes que fluctúan desde 0 (nunca) a 6 (siempre). Al analizar las propiedades de esta escala en Puerto Rico, Rodríguez Montalbán, Sánchez Cardona y 
Martínez Lugo (2014) encontraron un índice de consistencia interna alfa de Cronbach de .92.

\section{Resultados del Estudio 3}

Se realizó un análisis de correlación $r$ de Pearson entre las puntuaciones en la EAL y UWES-9, obteniéndose un coeficiente de $-.73 p$ $<.001$. Luego se realizó un análisis de correlación entre factores latentes con ecuaciones estructurales con partial least squares. En el mismo se obtuvo una correlación de $-.78 p<.001$. Cabe destacar que la correlación entre los factores latentes tiende a ser mayor debido a que las puntuaciones factoriales no contienen el error de medición (Chin \& Newsted, 1999).

Se evidencia validez discriminante para el constructo de aburrimiento laboral al correlacionarse con el engagement en el trabajo. Las correlaciones entre las variables son menores a .85 tal y como recomienda Brown (2015). La varianza compartida entre la EAL y UWES es de .61 lo que es inferior a los valores de varianza media extraída para ambos instrumentos tal y como recomiendan Fornell y Larcker (1981). En este estudio la varianza media extraída para EAL fue .76 y para UWES9 fue .66. Los valores alfa de Cronbach para las escalas fueron .97 para EAL y .93 para UWES-9. La fiabilidad compuesta para los factores latentes fue .97 para EAL y .95 para UWES-9.

\section{Discusión}

El objetivo principal de los estudios presentados fue desarrollar y analizar las propiedades psicométricas (validez de constructo, validez convergente, validez discriminante y fiabilidad) de la Escala de Aburrimiento Laboral (EAL) de Martínez-Lugo, Rodríguez-Montalbán y SánchezCardona. Basándonos en los resultados obtenidos, podemos concluir que la EAL es un instrumento válido y confiable para medir el aburrimiento en contextos laborales. La escala final posee 8 ítems, lo cual facilita su administración en las organizaciones y es una herramienta corta para fines investigativos y de diagnóstico.
La EAL posee excelentes propiedades psicométricas. Su coeficiente alfa de Cronbach excede el valor de .70 tal como lo recomiendan Nunnally y Bernstein (1994). De igual forma, la fiabilidad compuesta de la escala excede el valor de 70 tal como lo recomiendan Raykov y Shrout (2002). Cabe destacar que su confiabilidad se compara favorablemente con escalas como Lee Job Boredom Scale ( $\alpha$ : .95) (Vodanovich, 2003), Multidimensional State Boredom Scale ( $\alpha$ : .80, .89) (Alda et al., 2015; Vodanovich, 2003;) y Dutch Boredom Scale ( $\alpha: .80, .78)$ (Reijseger et al., 2012; van Wyk et al., 2016). El promedio de las correlacionesítem-total se compara favorablemente con las de Dutch Boredom Scale (.55) (Reijseger et al., 2012). Por último, el modelo unifactorial se ajusta mejor a los datos lo cual coincide con los resultados de Reijseger et al. (2012) y de van Wyk et al. (2016) referentes al Dutch Boredom Scale que también posee un factor.

Cabe destacar que la relación entre la EAL y la DUBS es muy alta. Esto evidencia que ambas escalas están midiendo el mismo constructo. Sin embargo, debemos destacar que la varianza media extraída de la EAL es mayor que la encontrada en DUBS. Esto evidencia que la EAL tiene menor error de medición, lo cual favorece las inferencias que se puedan realizar sobre el constructo.

\section{Implicaciones teóricas}

Al correlacionar el aburrimiento laboral con el engagement en el trabajo encontramos resultados similares a los obtenidos por Guglielmi et al. (2013) y por van Wyk et al. (2016). La literatura resalta que el engagement en el trabajo es considerado el opuesto teórico del síndrome de quemarse por el trabajo (burnout) (Salanova, Schaufeli, Llorens, Peiro \& Grau, 2000; Salanova \& Schaufeli, 2008; Schaufeli, Salanova, GonzálezRomá \& Bakker, 2002). Sin embargo, nuestros resultados evidencian una fuerte relación negativa entre el engagement y el aburrimiento, lo cual es cónsono con un planteamiento reciente de Schaufeli y Salanova (2014). Cabe destacar que este 
planteamiento teórico no había sido refrendado con evidencia empírica y nuestro estudio llena ese vacío. De acuerdo a Tabachnick y Fidell (2007) para establecer un opuesto empírico de un constructo es preciso obtener una correlación de -.80. En nuestro estudio encontramos una relación de -.78 entre el engagement y el aburrimiento. A pesar de no poder cumplir con lo recomendado por Tabachknick y Fidell, podemos concluir que una persona no puede estar engaged y aburrida en el trabajo a la misma vez, debido a que son dos experiencias psicológicas y estados motivacionales incompatibles. Mientras en el engagement se observa el vigor, la dedicación y el sentir que el tiempo pasa volando, en el aburrimiento observamos el desinterés, la desvinculación y la prolongación del tiempo.

\section{Implicaciones prácticas}

El desarrollo de la Escala de Aburrimiento Laboral (EAL) permitirá que los países de habla castellana contemos con una herramienta con las propiedades psicométricas adecuadas para poder analizar la variable aburrimiento en nuestros contextos laborales. Además, permitirá que podamos realizar investigaciones sobre dicha variable y cómo la misma se relaciona e incide en el comportamiento organizacional. Cabe destacar, que este instrumento es de fácil administración por ser breve y redactado en un lenguaje sencillo, lo cual permite que la escala sea útil para propósitos de investigación y de diagnóstico.

\section{Uso de la escala}

Dado a que el instrumento posee una estructura factorial unidimensional, se debe calcular una puntuación compuesta donde se promedian todos los ítems de la escala. Para ello se suman las puntuaciones obtenidas en cada ítem y se divide entre 8. Esto permitirá obtener una puntuación de aburrimiento que oscilará entre $0-6$.

\section{Limitaciones y fortalezas}

Los resultados de este trabajo deben ser interpretados de acuerdo a sus limitaciones, sin menospreciar sus aportaciones. La principal limitación que podemos identificar radica en que el muestreo de los tres estudios fue por disponibilidad, lo que no nos permite generalizar sus resultados. Sin embargo, la composición heterogénea de la muestra, así como la coincidencia de los resultados y la similitud de los hallazgos en los tres estudios donde utilizamos la EAL, podría aminorar esta limitación.

\section{Futuras líneas de investigación}

Entendemos que entre las posibles futuras líneas de investigación se encuentran analizar los antecedentes y las consecuencias de la experiencia del aburrimiento laboral. Del mismo modo, explorar los posibles factores psicosociales y organizacionales que pueden mitigar la experiencia del aburrimiento en el contexto laboral Latinoamericano.

\section{Conclusiones generales}

La psicología del trabajo tiene como finalidad entender los fenómenos que inciden en la vida de las personas en el entorno laboral. La situación de crisis económica y laboral actual obliga a muchas personas a aceptar trabajos que en muchas ocasiones no les permiten demostrar al máximo todo su potencial, llevándolos a experimentar el aburrimiento laboral. A través de los estudios presentados anteriormente logramos nuestro objetivo de desarrollar un instrumento en español con excelentes propiedades psicométricas que permite investigar el fenómeno del aburrimiento en el lugar de trabajo. Esperamos que las futuras investigaciones que se realicen permitan establecer una agenda común que incida en el desarrollo de la psicología del trabajo y las organizaciones en Latinoamérica y por ende, mejorar los ambientes de trabajo de las organizaciones en nuestros países. 


\section{Referencias}

Alda, M., Minguez, J., Montero-Marín, J., Gili, M., PueblaGuedea, M., Herrera-Mercadal, P., Navarro-Gil, M. \& García-Campayo, J. (2015). Validation of the Spanish version of the Multidimensional State Boredom Scale (MSBS). Health and Quality of Life Outcomes, 13:59. DOI 10.1186/s12955-015-0252-2

Baker, P. L. (1992). Bored and busy: Sociology of knowledge of clerical workers. Sociological Perspectives, 35(3), 489-503.

Brown, T.A. (2015). Confirmatory factor analysis for applied research (2nd Ed.). New York: Guildford Press.

Bruursema, K., (2007). How individual values and trait boredom interface with job characteristics and job boredom in their effects on counterproductive work behavior. Graduate Theses and Dissertations. http:// scholarcommons.usf.edu/etd/646

Bruursema, K., Kessler, S.R. \& Spector, P. (2011). Bored employees misbehaving: The relationship between boredom and counterproductive work behavior. Work \& Stress, 25(2), 93-107.

Chin, W. W. \& Newsted, P. R. (1999). Structural equation modeling analysis with small samples using partial least squares. In R. H. Hoyle (Ed.), Statistical strategies for small sample research (pp. 307-341). Thousand Oaks, CA: Sage.

Csikszentmihalyi, M. (1975). Beyond boredom and anxiety: Experiencing flow in work and play, San Francisco: Jossey-Bass.

Culp, N.A. (2006). The relationship of two facets of boredom proneness with the major dimensions of personality. Personality and Individual Differences, 41, 999-1007.

Fahlman, S., Mercer-Lynn, K., Flora, D. \& Eastwood, J. (2011). Development and validation of the Multidimensional State Boredom Scale. Assessment, 20, 68-85. doi: 10.1177/1073191111421303

Farmer, R. \& Sundberg, N.D. (1986). Boredom proneness: The development and correlates of a new scale. Journal of Personality Assessment, 50, 4-17.

Fisher, C.D. (1993). Boredom at work: A neglected concept. Human Relations, 46(3), 395-417).

Fornell, C., \& Larcker, D. F. (1981). Structural Equation Models with unobservable variables and measurement error: Algebra and statistics. Journal of Marketing Research, 18, 39-50.
Gemmill, G. \& Oakley, J. (1992). The meaning of boredom in organizational life. Group \& Organization Management, 17, 358-369.

González-Romá, V., Schaufeli, W. B., Bakker, A. B. \& Lloret, S. (2006). Burnout and work engagement: Independent factors or opposite poles? Jounal Journal of Vocational Behavior, 68, 65-174

Guglielmi, D., Simbula, S., Mazzetti, G., Tabanelli, M.C. \& Bonfiglioli, R. (2013). When the job is boring: The role of boredom in organizational contexts. Work, $45,311-322$.

Harju, L., Hakanen, J.J., \& Schaufeli, W.B. (2014). Job boredom and its correlates in 87 Finnish organizations. Journal of Occupational and Environmental Medicine, 56(9), 911-918.

Hill, A. B. (1975). Work variety and individual differences in occupational boredom. Journal of Applied Psychology, 60(1), 128-131.

Kass, S.J., Vodanovich, S.J., \& Callender, A. (2001). Statetrait boredom: Relationship

to absenteeism, tenure, and job satisfaction. Journal of Business and Psychology, 16,317-327.

Kline, T. J. B. (2005). Psychological testing: A practical approach to design and evaluation. Thousand Oaks: Sage Publications.

Locke, E. A., \& Bryan, J. F. (1967). Performance goals as determinants of level of performance and boredom. The Journal of Applied Psychology, 51(2), 120-130.

Loukidou, L., Loan-Clarke, J. \& Daniels, K. (2009). Boredom in the workplace: More than monotonous tasks. International Journal of Management Reviews, 11(4), 381-405.

Mann, S. (2012). Boredom at the checkout: Causes, coping strategies and outcomes of workplace boredom in a supermarket setting. Journal of Business and Retail Management Research, 6(2), 1-14.

Martin, M., Sadlo, G. \& Stew, G. (2006). The phenomenon of boredom. Qualitative Research in Psychology, 3, 193-211.

Mikulas, W. \& Vodanovich, S. (1993). The essence of boredom. The Psychological Record, 43, 3-12.

Nunnally, J.C. \& Bernstein, I.H. (1994). Psychometric theory. New York: McGraw-Hill.

O'Hanlon, J. (1981). Boredom: Practical consequences and a theory. Acta Psychologica, 49, 53-82. 
Piotrowski, C. (2013). Boredom research: an analysis of topical domain and historical trends. Journal of Instructional Psychology, 40 (2), 50-52.

Raykov, T., \& Shrout, P. E. (2002). Reliability of scales with general structure: Point and interval estimation using a structural equation modeling approach. Structural equation modeling, 9(2), 195-212.

Reijseger, G., Schaufeli, W., Peeters, M., Taris, T., van Beek, I. \& Ouweneel, E. (2012). Watching the paint dry at work: psychometric examination of the Dutch Boredom Scale. Anxiety, Stress \& Coping: An International Journal, 26(5), 508-525.

Rodríguez-Montalbán, R., Vélez-Pastrana, M. \& MeléndezSáez, M. (2012). El engagement y la interacción familia-trabajo: Un estudio exploratorio. Revista Interamericana de Psicología Ocupacional, 31(2), 7384.

Rodríguez-Montalbán, R., Martínez-Lugo, M., SánchezCardona, I. (2014). Análisis de las propiedades psicométricas de la Utrecht Work Engagement Scale en una muestra de trabajadores en Puerto Rico. Universitas Psychologica, 13(4), 1255-1266.

Salanova, M. \& Schaufeli, W.B. (2008). A cross-national study of work engagement as a mediator between job resources and proactive behaviour. The International Journal of Human Resource Management, 19, 116131.

Salanova, M., Schaufeli, W.B., Llorens, S., Peiro, J.M. \& Grau, R. (2000). Desde el 'burnout' al 'engagement': ¿Una nueva perspectiva? Revista de Psicología del Trabajo y las Organizaciones, 16, 117-134.

Schaufeli, W. B., Bakker, A. B., \& Salanova, M. (2006). The measurement of work engagement with a short questionnaire: A cross-national study. Educational and Psychological Measurement, 66(4), 701-716.

Schaufeli, W., \& Salanova, M. (2007). Work engagement: An emerging psychological concept and its implications for organizations. In S. W. Gilliland, D. D. Steiner, \& D. P. Skarlicki (Eds.), Research in social issues in management (Volume 5): Managing social and ethical issues in organizations (pp. 135-177). Greenwich, CT: Information Age Publishers.

Schaufeli, W. \& Salanova, M. (2014). Burnout, boredom and engagement. En M.C.W. Peeters, J. de Jonge \&
T.W. Taris. An introduction to contemporary work psychology (pp.293-320). New York: John Wiley \& Sons.

Schaufeli, W.B., Salanova, M., González-Romá, V., \& Bakker, A.B (2002). The measurement of engagement and burnout: A two sample confirmatory factor analytic approach. Journal of Happiness Studies, 3, 71-92.

Smith, M. (1944). Handbook of industrial psychology. New York: Philosophical Library.

Sohail, N., Ahmad, B., Tanveer, Y. \& Tarik, H. (2012). Workplace boredom among university faculty members in Pakistan. Interdisciplinary Journal of Contemporary Research in Business, 3(10), 919-925.

Tabachnick, B. G., \& Fidell, L. S. (2007). Using multivariate statistics. Boston, MA: Pearson Education.

van der Heijden, G., Schepers, J. \& Nijssen, E. (2012). Understanding workplace boredom among white collar employees: temporary reactions and individual differences. European Journal of Work and Organizational Psychology, 21(3), 349-375.

van Hooff, M.L.M. \& van Hooft, E.A.J. (2014). Boredom at work: proximal and distal consequences of affective work-related boredom. Journal of Occupational Health Psychology, 19(3), 348-359.

van Hooff, M.L.M. \& van Hooft, E.A.J. (2016). Workrelated boredom and depressed mood from a daily perspective: the moderating roles of work centrality and need satisfaction. Work \& Stress, 30(3), 209-227.

van Wyk, S.M., De Beer, L.T., Pienaar, J. \& Schufeli, W.B. (2016). The psychometric properties of a workplace boredom scale (DUBS) within the South African context. SA Journal of Industrial Psychology, 42(1), a1326.

Vodanovich, S. (2003). Psychometric measures of boredom: A review of the literature. The Journal of Psychology, 137(6), 569-595.

Vodanovich, S. \& Watt, J.D. (2016). Self-report measures of boredom: An updated review of the literature. The Journal of Psychology, 150(2), 196-228.

Watt, J.D. (2002). Fighting more than fires: Boredom proneness, workload stress, and underemployment among urban firefighters. Dissertation Abstracts International, 63, 2637. 


\section{Anexo A}

Escala de Aburrimiento Laboral (EAL) de 8 ítems, formato de respuesta de 0 (totalmente en desacuerdo) a 6 (totalmente de acuerdo), de Martínez-Lugo, Rodríguez-Montalbán y Sánchez-Cardona

\section{Dimensión 1}

Desvinculación

- Me siento aburrido en mi trabajo.

- Las tareas de mi trabajo me estimulan muy poco o nada.

- Siento que en mi trabajo estoy perdiendo tiempo, que podría estar utilizando en otras cosas.

- Las tareas que realizo en mi trabajo no me animan.

\section{Dimensión 2}

Desinterés

- Las tareas que realizo en mi trabajo NO me interesan, pero lo que hago me ayuda a pagar mis deudas.

- Las tareas que realizo en mi trabajo son irrelevantes para mí

\section{Dimensión 3}

Percepción de tiempo

- Las horas en mi trabajo se hacen largas porque NO tengo nada que hacer.

- $\quad$ Siento que el tiempo es eterno cuando estoy en mi trabajo. 